ate results. Without it you're more like someone taking pictures with a film camera: "You don't know how good the picture is until you have the film developed, but by then it might be too late, or you have too many pictures of the same thing," he says.

\section{Picking up the signal}

One application of Cellomics HCS systems is to study intracellular signalling pathways in oncology, as they enable a pathway to be 'walked' using multiplexing.

Another company pursuing ways to streamline signalling research is DiscoveRx of Fremont, California, which received a 2006 Frost \& Sullivan award for technology innovation for its PathHunter assay platform, which is used to study signalling pathways in intact cells. A recent addition to the range is a $\beta$-arrestin assay to study the activation of G-protein-coupled receptors (GPCRs), a common drug target.

An attractive feature of PathHunter assays is that they are relatively simple: they are adapted to microtitre plates, involve only one or two additions of reagents, and do not require cell washing or fixation. The assays are compatible with many different cell lines, especially those most commonly used in high-throughput screening, such as $\mathrm{CHO}$ and HEK cells.

PathHunter assays detect receptor activation by a technique called enzyme fragment complementation. Two modified $\beta$-galactoside fragments, fused to the receptor and the $\beta$-arrestin molecule, respectively, are brought together when $\beta$-arrestin binds to the acti- vated receptor and form an active enzyme that generates a chemiluminescent signal.

Discove Rx claims that PathHunter is the first chemiluminescence assay technology that can measure protein trafficking directly inside the cell - as such, it is especially suited to evaluating how cellular physiology is perturbed by drug candidates. It can be used to study translocation, degradation, secretion, proteinprotein interactions and membrane trafficking. The chemiluminescent signal generated does not need dedicated imaging technology and can be read in 96-, 384- or 1,536-well microplates with a standard luminometer.

Tecan's new Infinite series of microplate readers are compatible with its Freedom EVO liquid-handling workstation and are available as either filter- or monochrometer-based systems. According to Feiglin, the sensitivity of the luminescence readers makes them a popular choice for assays of GPCR activity. Using the 1,536-well optics options on the Infinite F500 filter-based microplate reader, a 1,536-well plate can be read in less than 30 seconds. Also new from Tecan is the HydroFlex automated microplate washing and vacuum filtration system, which can be integrated into the Freedom EVO platform.

CyBio of Jena, Germany, made its reputation as one of the first companies to offer multipipettors. It recently unveiled a new robotic liquid-handling platform, CyBiRoboSpense, which will work with almost any type of sample tube or plate, and can even transport plates to other instruments such as

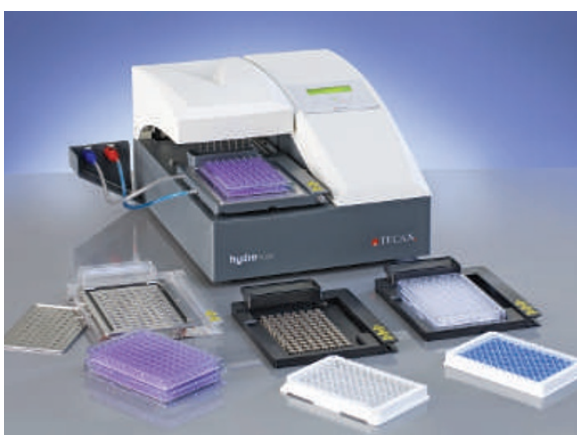

Tecan's HydroFlex platewasher can be integrated into the Freedom EVO system.

other pipettors, microplate readers or liquid chromatography or mass spectroscopy equipment. The CyBi-RoboSpense can also be used for complicated sample preparation and purification routines such as tryptic digests and magnetic bead separation.

\section{Microfluidics applications}

More companies are incorporating the principles of microfluidics into their instruments, making the movement of microlitre - or even nanolitre - volumes of solutions do what used to take hundreds of millilitres, not to mention a saving in time and labour. An innovator in this area is Gyros of Uppsala, Sweden, whose Gyrolab Bioaffy microlabs for protein purification and quantification are based on affinity-capture techniques. The Gyrolab Bioaffy 200 is ideal for high-sensitivity assays to detect low concentrations of proteins. It can be used, for example,

\title{
IN SILICO SCREENING WITH CHEMICAL INFORMATICS
}

Imagine evaluating the efficacy and safety of new drugs without having to lift a pipette. Advances in in silico drug development are beginning to make this possible.

The SYBYL range of

computational chemistry software from Tripos of St Louis, Missouri, will design compound libraries, select the best binding partners and screen potential drug molecules for safety and efficacy on the basis of known protein target and/or ligand structures - all in silico. A new advanced protein-modelling module predicts the structure of proteins of unknown structure using related proteins of known structure as the models. "It rapidly and accurately provides a valuable protein structure to be used for in silico docking experiments," says Kathleen Mensler, vice-president of marketing and corporate development at Tripos. Another new product, Surflex-Sim, helps find new classes of compounds that are structurally similar to already known, suboptimal, lead compounds, but that may be more active or safer.

Modelling software from Cresset BioMolecular Discovery in Letchworth, UK, uses surface properties or 'molecular fields' of proteins and other molecules to predict intermolecular interactions. "We know that structurally diverse compounds can bind to the same protein site and elicit the same response, but this has never been fully explained by molecular structure," says Sally Rose, Cresset's director of business development. "Our view is that molecular fields provide vital information for understanding and predicting activity," she says. The fields are derived by calculating the interaction energy of a 'probe' atom carrying a positive, negative or neutral charge with the compound. "Our fields summarize the key binding information, and we locate the most important regions around a ligand where the fields are strongest and binding is expected," says Rose. The original FieldScreen software for virtual screening has been joined by FieldTemplater, designed to find threedimensional bioactive conformations, and FieldAlign, a molecular-alignment tool for comparing the three-dimensional structures of two molecules. A pioneer of in silico modelling, De Novo Pharmaceuticals in Cambridge, UK, recently announced a new focus for its drug-discovery programme - finding drug targets in various metabolic diseases. De Novo has upgraded SkelGen, its proprietary

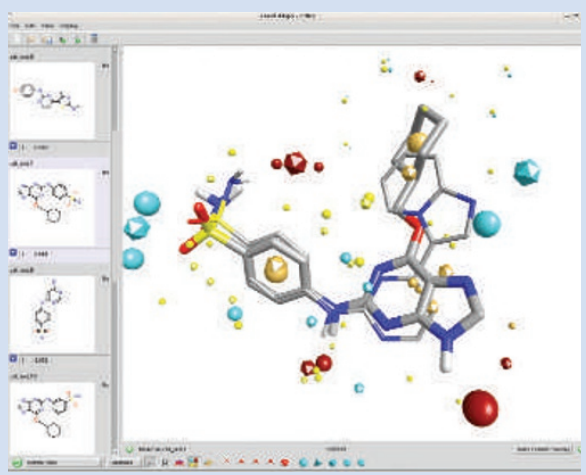

Line up: Cresset's FieldAlign program compares molecular structures. in silico drug-design platform, with the addition of Reflex, which takes into account the rotational flexibility of amino acids in the target's active site. This better evaluates the shape of the active site and its interaction with smallmolecule ligands, so that the modelling more closely resembles the dynamics of real protein-ligand interactions. 